\title{
On the nature of the neutrino
}

\author{
R. Romero* \\ Departamento de Física, División de Ciencias Básicas e Ingeniería, \\ Universidad Autónoma Metropolitana unidad Iztapalapa, San Rafael Atlixco $N^{\circ} 186$, \\ Col. Vicentina, C.P. 09340, Del. Iztapalapa, México D.F.
}

\begin{abstract}
Assuming that one neutrino type with definite mass is described by a massive Dirac field operator, it is shown that the physical one-particle states for particles and antiparticles can be rotated to each other, irrespective of their helicity. This result is used to prove that the neutrino must necessarily be a Majorana particle.

PACS numbers: 14.60.Lm, 11.10.-z, 11.30.Er

Keywords: Dirac neutrino, Majorana neutrino, momentum-dependent rotations.
\end{abstract}

\section{INTRODUCTION}

Originally conceived to be massless in the standard model, neutrinos are now known to oscillate in flavor and posses tiny but non-vanishing masses [1-3], although their absolute values are unknown, with data and estimations about squared-mass splittings and upper bounds for mass eigensates currently available [1]. Disregarding gravity, neutrinos interact only through the weak interaction, and only left-handed neutrinos and right-handed antineutrinos have ever been detected [1, 4].

One open problem in neutrino physics is the determination of their nature. Because they are electrically neutral two possibilities exist [5-10]: either neutrinos are distinct from their antiparticles and hence of the Dirac type (implying lepton number conservation), or they are equal to their antiparticles and hence of the Majorana type (implying lepton number violation). Theoretically, the latter is the preferred alternative for two main reasons: it provides a natural explanation for light neutrino masses through the see-saw mechanism [8], and allows for models of baryogenesis from leptogenesis that could explain the baryon asymmetry of the Universe [7, 1113]. Thus, in most extensions to the standard model it is $a b$ initio assumed that neutrinos are Majorana particles $[12,14]$.

When calculating amplitudes of neutrino processes it is found that, regardless of the process, the difference in matrix elements for Dirac and Majorana types is proportional to the ratio of the neutrino mass $m_{\nu}$ to its energy $E_{\nu}: m_{\nu} / E_{\nu}$, which becomes negligible small for high-energetic neutrinos with small masses [5]. This result makes very difficult the experimental determination of the nature of the neutrino, with the best option provided by neutrinoless double beta decay experiments, a process that can only occur if neutrinos are Majorana particles. The present experimental status in this regard is inconclusive [1], but there are both ongoing and planned experiments that could resolve the issue in a few years $[15,16]$.

In this letter I address the problem of the neutrino nature, from the perspective of canonical quantum field theory, and show that it must necessarily be of the Majorana type. This is done by assuming that a general neutrino with definite mass is described by a massive Dirac field operator and showing that, nonetheless, the physical one-particle states must be identified with the antiparticles ones because they can be rotated to each other, violating lepton number conservation in the process. I will also show that no such rotations are possible for massive Majorana field operators. The procedure involves momentum-dependent transformations acting on free field operators in an analogous way to the standard discrete transformations of parity and charge conjugation, with no models beyond the standard model assumed or required. Natural units with $\hbar=c=1$ and the Weyl representation of the gamma matrices

$$
\gamma^{0}=\left(\begin{array}{ll}
0 & 1 \\
1 & 0
\end{array}\right), \gamma=\left(\begin{array}{rr}
0 & \boldsymbol{\sigma} \\
-\boldsymbol{\sigma} & 0
\end{array}\right)
$$

here denoted in $2 \times 2$ block form, with $\boldsymbol{\sigma}=\left(\sigma^{1}, \sigma^{2}, \sigma^{3}\right)$ the standard Pauli matrices, will be used throughout.

\section{DIRAC CASE}

Let $a_{\lambda}(\mathbf{p})$ and $b_{\lambda}(\mathbf{p})$ respectively denote annihilation operators of Dirac particles and antiparticles of a given helicity $\lambda= \pm$, and $\hat{a}_{ \pm}(\mathbf{p})$ represent the corresponding operators for Majorana particles. In weak processes such as the decay of charged pions, the neutrinos produced can be scattered off a nuclear target resulting in a flux of muons. Neutrinos coming from $\pi^{+}$decay produce muons, while antineutrinos coming from $\pi^{-}$decay produce antimuons. Since neutrinos and antineutrinos have opposite helicities it is not possible to experimentally distinguish if the production of muons is due to lepton number conservation, in which case neutrinos are Dirac particles and $[6,17]$

$$
a(\mathbf{p}) \neq b(\mathbf{p})
$$


must hold irrespective of the helicity, or whether it is the helicity of the particles the responsible for the production, in which case neutrinos are Majorana particles and we must have

$$
\hat{a}_{+}(\mathbf{p}) \neq \hat{a}_{-}(\mathbf{p}) .
$$

The goal is to show that Eq. (3) is the only possibility, even if Eq. (2) is originally assumed. To this end let us consider one neutrino type of definite mass and assume it is a Dirac particle, described by a massive Dirac field. In the helicity basis, the field expansion is given by [18]

$$
\begin{array}{r}
\Psi(x)=\int \frac{d^{3} p}{(2 \pi)^{3}} \frac{1}{\sqrt{2 E_{\mathbf{p}}}} \sum_{\lambda= \pm}\left(u_{\lambda}(\mathbf{p}) a_{\lambda}(\mathbf{p}) e^{-i p . x}\right. \\
\left.+v_{\lambda}(\mathbf{p}) b_{\lambda}^{\dagger}(\mathbf{p}) e^{i p . x}\right),
\end{array}
$$

with the usual equal time anti-commutation relations satisfied by both field and operators [19]

$$
\begin{gathered}
\left\{\Psi_{\alpha}(\mathbf{x}), \Psi_{\beta}^{\dagger}(\mathbf{y})\right\}=\delta^{3}(\mathbf{x}-\mathbf{y}) \delta_{\alpha \beta} \\
\left\{\Psi_{\alpha}(\mathbf{x}), \Psi_{\beta}(\mathbf{y})\right\}=\left\{\Psi_{\alpha}^{\dagger}(\mathbf{x}), \Psi_{\beta}^{\dagger}(\mathbf{y})\right\}=0 \\
\left\{a_{\lambda}(\mathbf{p}), a_{\lambda^{\prime}}^{\dagger}(\mathbf{q})\right\}=\left\{b_{\lambda}(\mathbf{p}), b_{\lambda^{\prime}}^{\dagger}(\mathbf{q})\right\}=(2 \pi)^{3} \delta^{3}(\mathbf{p}-\mathbf{q}) \delta_{\lambda \lambda^{\prime}} .
\end{gathered}
$$

The positive- and negative-energy spinors $u_{\lambda}(\mathbf{p})$ and $v_{\lambda}(\mathbf{p})$ are four-component Dirac spinors in the helicity basis. Explicitly

$$
\begin{aligned}
& u_{\lambda}(\mathbf{p})=\left(\begin{array}{c}
\sqrt{E-\lambda|\mathbf{p}|} \xi_{\lambda}(\mathbf{p}) \\
\sqrt{E+\lambda|\mathbf{p}|} \xi_{\lambda}(\mathbf{p})
\end{array}\right), \\
& v_{\lambda}(\mathbf{p})=\left(\begin{array}{c}
-\lambda \sqrt{E+\lambda|\mathbf{p}|} \xi_{-\lambda}(\mathbf{p}) \\
\lambda \sqrt{E-\lambda|\mathbf{p}|} \xi_{-\lambda}(\mathbf{p})
\end{array}\right),
\end{aligned}
$$

with $\lambda= \pm$ and $\xi_{\lambda}$ two-component helicity spinors. Taking the three-momentum in spherical polar coordinates $\hat{\mathbf{p}}=(\sin \theta \cos \varphi, \sin \theta \sin \varphi, \cos \theta)$, solutions to the helicity eigenvalue equation $\boldsymbol{\sigma} \cdot \hat{\mathbf{p}} \xi_{\lambda}(\mathbf{p})=\lambda \xi_{\lambda}(\mathbf{p})$ are readily found

$$
\xi_{+}(\mathbf{p})=\left(\begin{array}{c}
\cos \left(\frac{\theta}{2}\right) \\
e^{i \varphi} \sin \left(\frac{\theta}{2}\right)
\end{array}\right), \xi_{-}(\mathbf{p})=\left(\begin{array}{c}
-e^{-i \varphi} \sin \left(\frac{\theta}{2}\right) \\
\cos \left(\frac{\theta}{2}\right)
\end{array}\right)
$$

Then it is straightforward to verify the following relations: $(\not p-m) u_{\lambda}(\mathbf{p})=0,(\not p+m) v_{\lambda}(\mathbf{p})=0$, with $\not p \equiv \gamma^{\mu} p_{\mu}$, and

$$
\begin{aligned}
& \bar{u}_{\lambda}(\mathbf{p}) u_{\lambda^{\prime}}(\mathbf{p})=2 m \delta_{\lambda, \lambda^{\prime}}, \\
& \bar{v}_{\lambda}(\mathbf{p}) v_{\lambda^{\prime}}(\mathbf{p})=-2 m \delta_{\lambda, \lambda^{\prime}} \\
& \bar{u}_{\lambda}(\mathbf{p}) v_{\lambda^{\prime}}(\mathbf{p})=0
\end{aligned}
$$

\begin{tabular}{|c|c|c|c|}
\hline State & Definition & Lepton number & Particle \\
\hline \hline$|\mathbf{p},-\rangle$ & $a_{-}^{\dagger}(\mathbf{p})|0\rangle$ & 1 & LH neutrino \\
\hline$|\overline{\mathbf{p}},+\rangle$ & $b_{+}^{\dagger}(\mathbf{p})|0\rangle$ & -1 & RH antineutrino \\
\hline$|\mathbf{p},+\rangle$ & $a_{+}^{\dagger}(\mathbf{p})|0\rangle$ & 1 & RH neutrino \\
\hline$|\overline{\mathbf{p}},-\rangle$ & $b_{-}^{\dagger}(\mathbf{p})|0\rangle$ & -1 & LH antineutrino \\
\hline
\end{tabular}

Table I. One particle states created from the vacuum by $\Psi$ and $\Psi^{\dagger}$. The states are assumed orthonormal and a possible normalization factor in the second column has been omitted. For neutrinos the $U(1)$ charge is associated with lepton number and not with electrical charge. In the case of states the over bar represents the antiparticle, and the terms left-handed $(\mathrm{LH})$ and right-handed $(\mathrm{RH})$ refer to $\mp$ helicity, respectively.

where the over bar represents the Dirac adjoint $\bar{u} \equiv u^{\dagger} \gamma^{0}$. Positive- and negative-energy spinors are also related to one another by charge conjugation

$$
\begin{aligned}
& i \gamma^{2} u_{\lambda}^{*}(\mathbf{p})=v_{\lambda}(\mathbf{p}), \\
& i \gamma^{2} v_{\lambda}^{*}(\mathbf{p})=u_{\lambda}(\mathbf{p}) .
\end{aligned}
$$

The one-particle states created by the field $\Psi$ in Eq. (4) and its Hermitian conjugate are summarized in Table 1, from them the following transformations are constructed

$$
\begin{aligned}
P & =\alpha_{1}|\mathbf{p},-\rangle\left\langle\mathbf{p},+\left|+\alpha_{2}\right| \mathbf{p},+\right\rangle\langle\mathbf{p},-| \\
& +\alpha_{3}|\overline{\mathbf{p}},-\rangle\left\langle\overline{\mathbf{p}},+\left|+\alpha_{4}\right| \overline{\mathbf{p}},+\right\rangle\langle\overline{\mathbf{p}},-|, \\
& \\
C & =\beta_{1}|\mathbf{p},-\rangle\left\langle\overline{\mathbf{p}},-\left|+\beta_{2}\right| \overline{\mathbf{p}},-\right\rangle\langle\mathbf{p},-| \\
& +\beta_{3}|\mathbf{p},+\rangle\left\langle\overline{\mathbf{p}},+\left|+\beta_{4}\right| \overline{\mathbf{p}},+\right\rangle\langle\mathbf{p},+|,
\end{aligned}
$$

with $\alpha_{i}, \beta_{i}, i=1, \ldots, 4$ complex coefficients of unit modulus $\left|\alpha_{i}\right|^{2}=\left|\beta_{i}\right|^{2}=1, i=1, \ldots, 4$, to assure unitarity. Applied to the states these transformations yield

$$
\begin{array}{ll}
P|\mathbf{p},-\rangle=\alpha_{2}|\mathbf{p},+\rangle, & C|\mathbf{p},-\rangle=\beta_{2}|\overline{\mathbf{p}},-\rangle \\
P|\mathbf{p},+\rangle=\alpha_{1}|\mathbf{p},-\rangle, & C|\mathbf{p},+\rangle=\beta_{4}|\overline{\mathbf{p}},+\rangle \\
P|\overline{\mathbf{p}},-\rangle=\alpha_{4}|\overline{\mathbf{p}},+\rangle, & C|\overline{\mathbf{p}},-\rangle=\beta_{1}|\mathbf{p},-\rangle, \\
P|\overline{\mathbf{p}},+\rangle=\alpha_{3}|\overline{\mathbf{p}},-\rangle, & C|\overline{\mathbf{p}},+\rangle=\beta_{3}|\mathbf{p},+\rangle .
\end{array}
$$

In the one-particle states basis, with rows $\{|\mathbf{p},-\rangle,|\mathbf{p},+\rangle,|\overline{\mathbf{p}},-\rangle,|\overline{\mathbf{p}},+\rangle\}$ and the corresponding bras as columns, explicit matrix representations of Eqs. (10) and (11) are given by

$$
P=\left(\begin{array}{cccc}
0 & \alpha_{1} & 0 & 0 \\
\alpha_{2} & 0 & 0 & 0 \\
0 & 0 & 0 & \alpha_{3} \\
0 & 0 & \alpha_{4} & 0
\end{array}\right), C=\left(\begin{array}{cccc}
0 & 0 & \beta_{1} & 0 \\
0 & 0 & 0 & \beta_{3} \\
\beta_{2} & 0 & 0 & 0 \\
0 & \beta_{4} & 0 & 0
\end{array}\right)
$$

Then the following properties are easily verified 


$$
\begin{gathered}
\operatorname{det} P=\alpha_{1} \alpha_{2} \alpha_{3} \alpha_{4}, \\
\operatorname{det} C=\beta_{1} \beta_{2} \beta_{3} \beta_{4}, \\
P P^{\dagger}=P^{\dagger} P=C C^{\dagger}=C^{\dagger} C=\mathbb{1}_{4} .
\end{gathered}
$$

Thus, the $P$ and $C$ transformations can be either rotations or reflections (respectively of \pm 1 determinant), depending on the choice of the $\alpha_{i}$ and $\beta_{i}$ phases. Assuming invariance of the vacuum under the transformations: $P, C|0\rangle=|0\rangle$, Eq. (12) yields

$$
\begin{array}{ll}
P a_{-}^{\dagger}(\mathbf{p}) P^{\dagger}=\alpha_{2} a_{+}^{\dagger}(\mathbf{p}), & C a_{-}^{\dagger}(\mathbf{p}) C^{\dagger}=\beta_{2} b_{-}^{\dagger}(\mathbf{p}), \\
P a_{+}^{\dagger}(\mathbf{p}) P^{\dagger}=\alpha_{1} a_{-}^{\dagger}(\mathbf{p}), & C a_{+}^{\dagger}(\mathbf{p}) C^{\dagger}=\beta_{4} b_{+}^{\dagger}(\mathbf{p}), \\
P b_{-}^{\dagger}(\mathbf{p}) P^{\dagger}=\alpha_{4} b_{+}^{\dagger}(\mathbf{p}), & C b_{-}^{\dagger}(\mathbf{p}) C^{\dagger}=\beta_{1} a_{-}^{\dagger}(\mathbf{p}), \\
P b_{+}^{\dagger}(\mathbf{p}) P^{\dagger}=\alpha_{3} b_{-}^{\dagger}(\mathbf{p}), & C b_{+}^{\dagger}(\mathbf{p}) C^{\dagger}=\beta_{3} a_{+}^{\dagger}(\mathbf{p}) .
\end{array}
$$

Up to phases, the $C$ transformation for operators in Eq. (15) coincide with the standard charge conjugation transformation $\mathcal{C}[19]$, since it exchanges particle and antiparticle without changing the helicity. But the $P$ transformation does not correspond to the standard parity transformation $\mathcal{P}$, even though it flips the helicity, because the latter requires $\mathcal{P} a_{\lambda}(\mathbf{p}) \mathcal{P}^{\dagger}=a_{-\lambda}(-\mathbf{p})$.

Equations (4) and (15) lead to

$$
\begin{array}{r}
P \Psi(x) P^{\dagger}=\int \frac{d^{3} p}{(2 \pi)^{3}} \frac{1}{\sqrt{2 E_{\mathbf{p}}}}\left(\alpha_{1}^{*} u_{+}(\mathbf{p}) a_{-}(\mathbf{p}) e^{-i p . x}\right. \\
+\alpha_{2}^{*} u_{-}(\mathbf{p}) a_{+}(\mathbf{p}) e^{-i p . x}+\alpha_{3} v_{+}(\mathbf{p}) b_{-}^{\dagger}(\mathbf{p}) e^{i p . x} \\
\left.+\alpha_{4} v_{-}(\mathbf{p}) b_{+}^{\dagger}(\mathbf{p}) e^{i p . x}\right), \quad
\end{array}
$$

and, in order for this to represent a transformation of the field, we need to make a consistent choice for the $\alpha_{i}$ coefficients and flip the helicity of the spinors using some unitary matrix. The latter can be achieved by momentumdependent transformations, built from the spinors themselves in analogy with Eq. (10). One such transformation is given by

$$
\begin{gathered}
R(\mathbf{p})=-i \cos (\varphi) \sin (\theta) \gamma^{0} \gamma^{1} \gamma^{2} \\
+i \sin ^{2}\left(\frac{\theta}{2}\right) \sin (2 \varphi) \gamma^{0} \gamma^{1} \gamma^{3} \\
+i\left(\cos ^{2}\left(\frac{\theta}{2}\right)-2 \cos (2 \varphi) \sin ^{2}\left(\frac{\theta}{2}\right)\right) \gamma^{0} \gamma^{2} \gamma^{3}
\end{gathered}
$$

which is unitary and of unit determinant, hence a rotation. In terms of the spinors it reads

$$
\begin{aligned}
R(\mathbf{p}) & =\frac{1}{2 m}\left(u_{+}(\mathbf{p}) \bar{u}_{-}(\mathbf{p})+u_{-}(\mathbf{p}) \bar{u}_{+}(\mathbf{p})\right. \\
& \left.-v_{+}(\mathbf{p}) \bar{v}_{-}(\mathbf{p})-v_{-}(\mathbf{p}) \bar{v}_{+}(\mathbf{p})\right)
\end{aligned}
$$

from which, together with Eq. (8), it is directly verified that

$$
\begin{aligned}
R(\mathbf{p}) u_{\lambda}(\mathbf{p}) & =u_{-\lambda}(\mathbf{p}), \\
R(\mathbf{p}) v_{\lambda}(\mathbf{p}) & =v_{-\lambda}(\mathbf{p}) .
\end{aligned}
$$

Thus, Eqs. (16) and (19) lead to

$$
P \Psi(x) P^{\dagger}=R(\mathbf{p}) \Psi(x),
$$

provided that

$$
\alpha_{i}=1, i=1, \ldots, 4
$$

which in turn, from Eq. (14), makes $P$ a rotation. If the $\hat{\mathbf{z}}$ axis is taken as the quantization axis, as is commonly chosen, we can take $\theta=\varphi=0$ and then the spinors in Eq. (7) reduce to eigenstates of $\sigma^{3}$ and, from Eq. (17), the matrix $R(\mathbf{p})$ reduces to $i \gamma^{0} \gamma^{2} \gamma^{3}$.

Let us now turn to the $C$ transformation, Eqs. (4) and (15) yield

$$
\begin{array}{r}
C \Psi(x) C^{\dagger}=\int \frac{d^{3} p}{(2 \pi)^{3}} \frac{1}{\sqrt{2 E_{\mathbf{p}}}}\left(\beta_{4}^{*} u_{+}(\mathbf{p}) b_{+}(\mathbf{p}) e^{-i p \cdot x}\right. \\
+\beta_{2}^{*} u_{-}(\mathbf{p}) b_{-}(\mathbf{p}) e^{-i p \cdot x}+\beta_{3} v_{+}(\mathbf{p}) a_{+}^{\dagger}(\mathbf{p}) e^{i p \cdot x} \\
\left.+\beta_{1} v_{-}(\mathbf{p}) a_{-}^{\dagger}(\mathbf{p}) e^{i p \cdot x}\right) \cdot
\end{array}
$$

Then, using Eq. (9) and choosing the phases

$$
\beta_{i}=1, i=1, \ldots, 4
$$

the field transformation is

$$
C \Psi(x) C^{\dagger}=i \gamma^{2} \Psi^{*}(x)
$$

The matrix $i \gamma^{2}$ is a rotation, and from Eqs. (14) and (23) we get that $C$ is also a rotation. Furthermore, its action on the field is lineal, even though the complex conjugate field appears in Eq. (24). This implies that charge conjugation can be consistently implemented as a rotation, just as in the case of the $P$ transformation.

Already from Eqs. (14), (15), and (23) we get that particles and antiparticles of the same helicity can be rotated to each other and so they must be identified $a_{\lambda}(\mathbf{p})=b_{\lambda}(\mathbf{p})$, in contradiction with Eq. (2). However, weak interactions involve left-handed neutrinos and right-handed antineutrinos, meaning we really ought to compare $a$ 's and $b$ 's of different helicities. But first let us check the commutation relations between the $C$ transformation and the Hamiltonian and lepton number operators. Following the usual procedure [19] and using Eqs. (4) and (5) we obtain 


$$
\begin{aligned}
\mathcal{H} & =\int d^{3} x \bar{\Psi}(-i \boldsymbol{\gamma} \cdot \nabla+m) \Psi \\
& =\int \frac{d^{3} p}{(2 \pi)^{3}} \sum_{\lambda= \pm} E_{\mathbf{p}}\left(a_{\lambda}^{\dagger}(\mathbf{p}) a_{\lambda}(\mathbf{p})+b_{\lambda}^{\dagger}(\mathbf{p}) b_{\lambda}(\mathbf{p})\right),
\end{aligned}
$$

$$
\begin{aligned}
L & =\int d^{3} x \Psi^{\dagger}(x) \Psi(x) \\
& =\int \frac{d^{3} p}{(2 \pi)^{3}} \sum_{\lambda= \pm}\left(a_{\lambda}^{\dagger}(\mathbf{p}) a_{\lambda}(\mathbf{p})-b_{\lambda}^{\dagger}(\mathbf{p}) b_{\lambda}(\mathbf{p})\right) .
\end{aligned}
$$

The operator in Eq. (26) is the charge operator for charged fermions, but for neutrinos it acts as a lepton number operator $L$ [9]. Then it follows from Eqs. (15), (25), and (26) that

$$
[\mathcal{H}, L]=[\mathcal{H}, C]=\{L, C\}=0,
$$

where the square and curly brackets respectively represent the commutator and the anti-commutator. These relations imply that the physical states can either be eigenstates of $\mathcal{H}$ and $L$ or of $\mathcal{H}$ and $C$. But the action of the $C$ rotation means that lepton number conservation is violated even if the former alternative is chosen, leading to neutrino states effectively behaving as Majorana particles.

Let us now consider the combined action of the $P$ and $C$ transformations. Using the phase conventions of Eqs. (21) and (23) we get from Eqs. (14) and (15) that $C P$ is a rotation that changes a particle into the antiparticle of opposite helicity, and from Eqs. (9) and (19) the field transformation is

$$
C P \Psi(x)(C P)^{\dagger}=i \gamma^{2} R^{*}(\mathbf{p}) \Psi^{*}(x),
$$

and it can be verified that the combined $C P$ transformation also satisfies Eq. (5). The $C P$ transformation implies that opposite helicity particles and antiparticles can be rotated to each other, and so they must be identified, again in contradiction with Eq. (2). Thus, we have proved that Eq. (2) is untenable in general, leaving Eq. (3) as the only possibility, and it remains to show that this is indeed the case.

\section{MAJORANA CASE}

The Majorana field expansion is obtained directly from Eq. (4), with $a_{\lambda}(\mathbf{p})=b_{\lambda}(\mathbf{p}) \equiv \hat{a}_{\lambda}(\mathbf{p})$

$$
\begin{array}{r}
\nu(x)=\int \frac{d^{3} p}{(2 \pi)^{3}} \frac{1}{\sqrt{2 E_{\mathbf{p}}}} \sum_{\lambda= \pm}\left(u_{\lambda}(\mathbf{p}) \hat{a}_{\lambda}(\mathbf{p}) e^{-i p \cdot x}\right. \\
\left.+\zeta v_{\lambda}(\mathbf{p}) \hat{a}_{\lambda}^{\dagger}(\mathbf{p}) e^{i p . x}\right)
\end{array}
$$

where, for generality sake, a creation phase $\zeta$ has been introduced [5]. The field satisfies the Majorana condition $\nu(x)=i \zeta^{*} \gamma^{2} \nu^{*}(x)$. Since now there are only two oneparticle states available, the ones created off the vacuum by $\hat{a}_{+}^{\dagger}(\mathbf{p})$ and $\hat{a}_{-}^{\dagger}(\mathbf{p})$, there is only one type of transformation connecting states of opposite helicity. Denoting it by $\Omega$, it is given by

$$
\Omega=\delta_{1}|\mathbf{p},-\rangle\left\langle\mathbf{p},+\left|+\delta_{2}\right| \mathbf{p},+\right\rangle\langle\mathbf{p},-|=\left(\begin{array}{cc}
0 & \delta_{2} \\
\delta_{1} & 0
\end{array}\right),
$$

where $\delta_{1,2}$ are phases, the states are now $|\mathbf{p}, \pm\rangle=$ $\hat{a}_{ \pm}^{\dagger}(\mathbf{p})|0\rangle$, and the matrix form in the last equality is obtained in this basis. It follows that

$$
\Omega \Omega^{\dagger}=\Omega^{\dagger} \Omega=\mathbb{1}_{2}, \operatorname{det} \Omega=-\delta_{1} \delta_{2} .
$$

In order for $\Omega$ to be a rotation we must have $\delta_{1}=-1 / \delta_{2}$, and so we can generally write

$$
\delta_{1}=-\delta_{2}^{*}=e^{i \omega},
$$

with $\omega$ a real parameter. For the operators, and again assuming invariance of the vacuum, Eqs. (30) and (32) imply

$$
\Omega \hat{a}_{+}^{\dagger}(\mathbf{p}) \Omega^{\dagger}=e^{i \omega} \hat{a}_{-}^{\dagger}(\mathbf{p}), \Omega \hat{a}_{-}^{\dagger}(\mathbf{p}) \Omega^{\dagger}=-e^{-i \omega} \hat{a}_{+}^{\dagger}(\mathbf{p})
$$

which yields, upon applying it to the Majorana field in Eq. (29)

$$
\begin{aligned}
& \Omega \nu(x) \Omega^{\dagger}=\int \frac{d^{3} p}{(2 \pi)^{3}} \frac{1}{\sqrt{2 E_{\mathbf{p}}}}\left(e^{-i \omega} u_{+}(\mathbf{p}) \hat{a}_{-}(\mathbf{p}) e^{-i p . x}\right. \\
&-e^{i \omega} u_{-}(\mathbf{p}) \hat{a}_{+}(\mathbf{p}) e^{-i p . x}+\zeta e^{i \omega} v_{+}(\mathbf{p}) \hat{a}_{-}^{\dagger}(\mathbf{p}) e^{i p . x} \\
&\left.-\zeta e^{-i \omega} v_{-}(\mathbf{p}) \hat{a}_{+}^{\dagger}(\mathbf{p}) e^{i p . x}\right) .
\end{aligned}
$$

This last result is analogous to the one in Eq. (16), therefore we need a unitary matrix $\hat{R}$ acting on the spinors, as in Eq. (19), that flips the helicity and provides the required phases. Allowing for arbitrary phases in the expansion of Eq. (18) provides the most general transformation between different helicity spinors of the same energy. Thus, a suitable modification of Eq. (18) accomplishes the required transformation, this is

$$
\begin{aligned}
\hat{R}(\mathbf{p}) & =\frac{1}{2 m}\left(e^{-i \omega} u_{+}(\mathbf{p}) \bar{u}_{-}(\mathbf{p})-e^{i \omega} u_{-}(\mathbf{p}) \bar{u}_{+}(\mathbf{p})\right. \\
& \left.-e^{i \omega} v_{+}(\mathbf{p}) \bar{v}_{-}(\mathbf{p})+e^{-i \omega} v_{-}(\mathbf{p}) \bar{v}_{+}(\mathbf{p})\right)
\end{aligned}
$$


but at the cost of loosing unitarity, since now $\hat{R}^{\dagger}(\mathbf{p}) \hat{R}(\mathbf{p}) \neq \mathbb{1}_{4}$ as is readily verified. This result shows that it is not possible to consistently make $\Omega$ a rotation of the Majorana field, which implies that the operators $\hat{a}_{\lambda}(\mathbf{p})$ cannot be rotated to each other, and so Eq. (3) must hold. The task is now complete and it is concluded that the neutrino is a Majorana particle.

\section{CONCLUDING REMARKS}

The arguments presented in this letter, showing the Majorana nature of the neutrino, are ultimately related to the degrees of freedom of Dirac and Majorana particles. It is the fact that a Dirac particle possesses four degrees of freedom which allows for the existence of the $C$ and $P$ transformations, as expressed in Eqs. (10) and (11), which lead to the identification of neutrino and antineutrino states, in direct contradiction to the fact that they should be different, and to lepton number violation. It is also the fact that a Majorana particle has only two degrees of freedom which prevents a similar contradiction to arise if the neutrino is assumed to be a Majorana particle, leaving such an assumption as the only viable possibility. These considerations also show that the conclusion reached is fundamental, and must hold independently of the mass generation, oscillation, and mixing mechanisms, all of which cannot alter the fundamental degrees of freedom. The final vindication of the Majorana nature of the neutrino must come from the experimental confirmation of neutrinoless double beta decays.

As a final note, Eqs. (24) and (28) also imply that charge conservation is violated for free charged fermions or, equivalently, that free fermions are essentially Majorana particles, irrespective of their charge. This, of course, no longer holds for interactive charged fermions, since the operators $C$ and $C P$ do not commute with the current operator $\hat{J}^{\mu}$ defined in the usual way [19]. These results could be relevant in explaining the Universe baryon asymmetry, a possibility that calls for further investigation.

* ricardo.romero@xanum.uam.mx
[1] K. A. Olive et al. (Particle Data Group), Chin. Phys. C38, 090001 (2014).

[2] S. M. Bilenky, Phys. Part. Nucl. 46, 475 (2015), arXiv:1501.00232 [hep-ph].

[3] T. Kajita, International Journal of Modern Physics A 24, 3437 (2009).

[4] M. Goldhaber, L. Grodzins, and A. W. Sunyar, Phys. Rev. 109, 1015 (1958).

[5] R. N. Mohapatra and P. B. Pal, Massive Neutrinos in Physics and Astrophysics; 3rd ed., World Scientific Lecture Notes in Physics (World Scientific, Singapore, 2004).

[6] M. Zralek, Acta Phys.Polon. B28, 2225 (1997), arXiv:hep-ph/9711506 [hep-ph].

[7] M. Fukugita and T. Yanagida, Physics of Neutrinos: and Application to Astrophysics, Theoretical and Mathematical Physics (Springer Berlin Heidelberg, 2003).

[8] S. F. King, Reports on Progress in Physics 67, 107 (2004).

[9] C. Giunti and C. Kim, Fundamentals of Neutrino Physics and Astrophysics (OUP Oxford, 2007).

[10] S. T. Petcov, Adv. High Energy Phys. 2013, 852987 (2013), arXiv:1303.5819 [hep-ph].

[11] P. D. Bari, Contemporary Physics 53, 315 (2012), http://dx.doi.org/10.1080/00107514.2012.701096.

[12] E. Akhmedov, in The Physics of Ettore Majorana (Cambridge University Press, 2014) pp. 303-353, cambridge Books Online, arXiv:1412.3320 [hep-ph].

[13] S. T. Petcov, International Journal of Modern Physics A 29, 1430028 (2014).

[14] R. Mohapatra et al., Rept. Prog. Phys. 70, 1757 (2007), arXiv:hep-ph/0510213.

[15] S. M. Bilenky and C. Giunti, Mod. Phys. Lett. A27, 1230015 (2012), arXiv:1203.5250 [hep-ph].

[16] W. Maneschg, Nuclear and Particle Physics Proceedings 260, 188 (2015), the 13th International Workshop on Tau Lepton Physics (Tau2014).

[17] M. Czakon, M. Zralek, and J. Gluza, Recent developments in theory of fundamental interactions. Proceedings, 23rd School of Theoretical Physics, Ustron, Poland, September 15-22, 1999, Acta Phys. Polon. B30, 3121 (1999), arXiv:hep-ph/9910357 [hep-ph].

[18] A. Duncan, The Conceptual Framework of Quantum Field Theory (OUP Oxford, 2012).

[19] M. Peskin and D. Schroeder, An introduction to quantum field theory, Advanced book program (Addison-Wesley Pub. Co., 1995). 